\title{
The Effects of Metallicity and Grain Growth and Settling on the Early Evolution of Gaseous Protoplanets
}

\author{
R. Helled ${ }^{1}$ and P. Bodenheimer ${ }^{2}$ \\ ${ }^{1}$ Department of Earth and Space Sciences, \\ University of California, Los Angeles, CA 90095 1567, USA \\ ${ }^{2}$ Department of Astronomy and Astrophysics, \\ UCO/ Lick Observatory, University of California, Santa Cruz, \\ CA 95064, USA \\ rhelled@ucla.edu (R. H.); peter@ucolick.org(P. B.)
}

\begin{abstract}
Giant protoplanets formed by gravitational instability in the outer regions of circumstellar disks go through an early phase of quasi-static contraction during which radii are large $(\sim 1 \mathrm{AU})$ and internal temperatures are low $(<2000 \mathrm{~K})$. The main source of opacity in these objects is dust grains. We investigate two problems involving the effect of opacity on the evolution of isolated, non-accreting planets of 3, 5, and $7 \mathrm{M}_{J}$. First, we pick three different overall metallicities for the planet and simply scale the opacity accordingly. We show that higher metallicity results in slower contraction as a result of higher opacity. It is found that the pre-collapse time scale is proportional to the metallicity. In this scenario, survival of giant planets formed by gravitational instability is predicted to be more likely around lowmetallicity stars, since they evolve to the point of collapse to small size on shorter time scales. But metalrich planets, as a result of longer contraction times, have the best opportunity to capture planetesimals and form heavy-element cores. Second, we investigate the effects of opacity reduction as a result of grain growth and settling, for the same three planetary masses and for three different values of overall metallicity. When these processes are included, the pre-collapse time scale is found to be of order 1000 years for the three masses, significantly shorter than the time scale calculated without these effects. In this case the time scale is found to be relatively insensitive to planetary mass and composition. However, the effects of planetary rotation and accretion of gas and dust, which could increase the timescale, are not included in the calculation. The short time scale we find would preclude metal enrichment by planetesimal capture, as well as heavy-element core formation, over a large range of planetary masses and metallicities.
\end{abstract}




\section{Introduction}

One suggested mechanism for giant planet formation is gravitational (disk) instability in which giant planets are formed by fragmentation in the protoplanetary disk (e.g., Cameron, 1978; Boss, 1997). The standard model for giant planet formation, however, is core accretion, in which the heavy-element core forms first by accretion of planetesimals and later captures a gaseous envelope (e.g., Pollack et al., 1996; Hubickyj et al., 2005). While solar-system-like giant planets are most likely to be formed by the core accretion mechanism, the disk instability model may be responsible for the formation of massive gaseous planets at very large radial distances (e.g., Rafikov, 2007; Cai et al., 2009). Giant planets such as those detected recently by direct imaging (Kalas et al., 2008; Marois et al., 2008) cannot easily be explained with the standard core accretion model due to the extremely long core formation timescales at large distances from the star (e.g., Dodson-Robinson et al., 2009). On the other hand, theoretical work suggests that gravitational instabilities are likely to occur at radial distances beyond 50 or even 100 AU (Boley, 2009; Rafikov, 2009), supporting the idea that massive gaseous planets on wide orbits may form by that mechanism.

Generally speaking, the main weakness of the gravitational instability model for giant planet formation is the uncertainty regarding whether clumps that are formed in the disk can actually survive and evolve to become gravitationally bound planets. While many of the numerical simulations have found disks that are gravitationally unstable under given conditions and have detected formation of clumps, only in a few cases have the objects been found to survive on longer timescales (see review by Durisen et al., 2007). Much work has been devoted to the question of whether clumps can cool fast enough to survive tidal disruption (e.g., Boss, 2008; Rafikov, 2007). Currently, due to limited resolution and computer power, three-dimensional hydrodynamic models cannot closely follow the evolution of the forming clumps long enough to verify whether these objects can indeed survive.

The evolution of protoplanets formed by gravitational instabilities includes three main stages. During the first stage, the newly-formed planetary object is cold and extended with a radius of a few thousand times Jupiter's present radius $\left(\mathrm{R}_{J}\right)$, with hydrogen being in molecular form $\left(\mathrm{H}_{2}\right)$. The clump contracts quasistatically on time scales of $10^{4}-10^{6}$ years, depending on mass, and as it contracts its internal temperatures increase. Once a central temperature of $\sim 2000 \mathrm{~K}$ is reached, the molecular hydrogen dissociates, and a dynamical collapse of the entire protoplanet occurs (the second stage). The extended phase is known as the 'pre-collapse stage' (Decampli and Cameron, 1979), and during that phase the object is at most risk to be destroyed by tidal disruption and disk interactions. After the dynamical collapse the planet becomes compact and dense, with the radius being a few times $\mathrm{R}_{J}$. During this third stage it is therefore less likely to be disrupted, although the planetary object still has the danger of falling into its parent star due to inward migration. The protoplanet then continues to cool and contract on a much longer timescale ( $10^{9}$ years).

During the extended state the protoplanet is most vulnerable to disk torques and tidal encounters, and 
therefore its survival is questionable. Shortening the pre-collapse timescale may reduce the risk of disruption since the transition to a "point-like" (gravitationally bound) protoplanet occurs faster. The time scale of the pre-collapse stage is also important with respect to the final planetary composition and structure. During the extended phase the protoplanet can capture heavy elements in the form of planetesimals and enrich the interior with these elements (e.g., Helled et al., 2006). Cores can be formed in these objects by settling of heavy elements to the planetary center as long as the internal temperatures are low (e.g., Helled et al., 2008). As a result, a longer extended phase could lead to further enrichment with heavy elements and would provide more time for forming cores. The more massive the protoplanet, the shorter the pre-collapse timescale (Helled and Bodenheimer, 2010, hereafter paper I). This may imply that in the disk instability scenario more massive planetary objects are likely to survive while lower mass planets (if they survive) will tend to be enriched with heavy elements and contain cores.

An important parameter that controls the contraction timescale is the planetary opacity. Lower opacity leads to faster cooling and therefore shortens the pre-collapse phase (e.g., Pollack et al., 1996). Below we investigate the effects of opacity reduction due to different metallicities (section 2) and grain growth and sedimentation (section 3) on the planetary evolution. The results are discussed in section 4 and the conclusions summarized in section 5 .

\section{Planetary Metallicity}

The question of whether stellar (and disk) metallicity affects the frequency of giant planet formation by gravitational instabilities has been investigated by different authors. Boss (2002) varied the assumed opacity in the models by factors of 10 and 0.1 , and found little difference in the results. As a result, Boss (2002) suggested that gravitational collapse is insensitive to the system's metallicity. Cai et al. (2006a,b) investigated the sensitivity to opacity in their models, but found that changing the opacity by factors of 0.25 to 2 led to enhanced fragmentation when the opacity was lowered. Meru and Bate (2010) also find, from three-dimensional radiation-hydrodynamic simulations, that low disk opacity tends to allow the disk to cool faster and promotes fragmentation (Gammie, 2001); therefore lower metallicity is more likely to result in fragmentation. Finally, Mayer et al. (2007) found enhanced fragmentation when the mean molecular weight $\mu$ was increased, but required rather large increases in $\mu$ (with no change of opacity), from 2.4 to 2.7 , to make a significant effect, and concluded that increasing metallicity would result in more fragmentation. All these groups reached different conclusions in some sense, though it should be noticed that the investigations were somewhat different. At present, the topic of fragmentation's sensitivity to metallicity is still unsolved.

Thus, metallicity can also be significant in the context of the protoplanets' evolution. Planetary metallicity (opacity) has a fundamental role in governing the cooling timescale of the planetary object and therefore the protoplanet's contraction. As a result, metallicity has a direct impact on the pre-collapse evolution of 
the newly-formed planets, and possibly their survival.

In this section we present the pre-collapse evolution (ending when central temperatures reach $\sim 2000 \mathrm{~K}$ ) for different planetary masses assuming different metallicities. Extrasolar planets have been detected around stars with very different metallicities, with the metallicity of the parent stars ranging between $[\mathrm{Fe} / \mathrm{H}] \sim-1$ and $[\mathrm{Fe} / \mathrm{H}] \sim+0.5$. Here, to model protoplanets with different metallicities, we simply multiply the grain opacity, calculated with an interstellar size distribution and solar abundances, by the corresponding metallicity. The Rosseland mean opacities are obtained from Pollack et al. (1985) and Alexander and Ferguson (1994). Thus, to model the evolution of a planetary object with $[\mathrm{Fe} / \mathrm{H}]=-0.477$, we multiply the solar grain opacity by a factor of $1 / 3$ everywhere. The planets are assumed to have solar abundances of hydrogen and helium; the equation of state is essentially an ideal gas of neutral He and molecular H. The standard stellar structure equations are solved (Bodenheimer et al. 1980), with surface boundary conditions appropriate for a gray photosphere

$$
L=4 \pi R^{2} \sigma_{B} T_{\text {eff }}^{4} \quad \text { and } \quad \kappa_{R} P=\frac{2}{3} g
$$

where $L$ is the total luminosity, $R$ is the outer radius, $\sigma_{B}$ is the Stefan-Boltzmann constant, $T_{\text {eff }}$ is the surface temperature, and $g, P$, and $\kappa_{R}$ are, respectively, the acceleration of gravity, the pressure, and the Rosseland mean grain opacity at the surface.

The initial radii of the protoplanets were chosen to fall inside the tidal radius at $20 \mathrm{AU}$ for a $3 \mathrm{M}_{J}$ protoplanet. Since all of the planets have an initial radius of $\sim 2 \mathrm{AU}$ they all fall within the Hill radius at distances larger than $20 \mathrm{AU}$. At smaller radial distances, planets could be tidally disrupted. Our initial radii are in good agreement with 3D numerical simulations of clump formation in gravitationally unstable disks (Boley et al., 2010).

Figures 1-3 present the evolutionary tracks for three planetary masses: 3,5 and $7 \mathrm{M}_{J}$, with solar, $3 \times$ solar and, solar $/ 3$ compositions $([\mathrm{Fe} / \mathrm{H}]=0,+0.477$, and -0.477 , respectively). More massive protoplanets have shorter pre-collapse evolutions. Protoplanets with similar masses but higher metallicities have longer evolutionary paths due to the higher opacity that results in slower energy loss. Objects with low metallicities on the other hand, have short pre-collapse timescales due to their ability to radiate energy efficiently (lower opacities), and therefore contract on shorter timescales. The results suggest that a reduction of the opacity everywhere by a factor of $\sim 3$ compared to solar abundance results in a pre-collapse phase shorter by a similar factor. In a similar way, increasing the metallicity by a factor of three results in an evolutionary timescale that is longer by about a factor of three. During the pre-collapse stage the protoplanets behave nearly as polytropes, and the radius is scaled inversely to the central temperature. Since the opacity for metal-rich planets is higher, for a similar radius the luminosity (as well as effective temperature) will be lower for metal-rich objects. Metal-poor protoplanets have higher luminosities and effective temperatures for a similar radius. 
A planetary object of three Jupiter masses can contract for $\sim 2 \times 10^{5}$ years before it reaches central temperatures of $2000 \mathrm{~K}$, if its composition is three times solar. Such a slow contraction could support the possibility of core formation and additional enrichment in heavy elements via planetesimal capture (e.g., Helled et al., 2006, 2008). These processes, which can take place during the precollapse evolution, can affect the final compositions and internal structures of the planetary objects. The analysis suggests that the metallicity (opacity) of the protoplanet (and therefore stellar metallicity) has an important impact on its evolution. Metal-rich objects have a better opportunity to capture additional solids and form heavy-element cores than metal-poor objects. On the other hand, the result may lead to the conclusion that survival of giant planets formed via gravitational instability is more likely to occur around low metallicity stars due to the shorter pre-collapse timescale, which minimizes the period in which the protoplanets are most vulnerable for destruction. However, this conclusion is based on the assumption that the grains in the planet remain well mixed throughout the interior and do not change their size distribution during the evolution. In fact, grains can grow, and settle, resulting in a reduction of the planetary opacity (e.g., Podolak, 2003; Helled et al., 2008; Movshovitz and Podolak, 2008). This possibility is investigated in the next section.

\section{Opacity Reduction Caused by Grain Coagulation and Sedimen- tation}

As protoplanets contract, grains in their atmospheres can grow, settle, and reduce the planetary opacity (Podolak, 2003). The radiative thin outer region of the protoplanet is of primary interest, since this region essentially controls the cooling of the planet. The evolutionary calculations described in the previous section show that most of the interior, below the radiative outer layer, is convective. To model grain coagulation and sedimentation and to compute the effect on the planetary opacity we use a numerical procedure recently presented by Movshovitz and Podolak (2008), kindly provided by N. Movshovitz. A detailed description of the calculation can be found in Movshovitz and Podolak (2008) and references therein. Below we briefly discuss the physics behind the numerical procedure.

At each level $r$ in the protoplanet at time $t$ the number density of grains with mass between $m$ and $m+d m$ is given by $n(m, r, t) d m$. The evolution of the grain distribution due to collisions is described by the Smoluchowski equation

$$
\begin{gathered}
\frac{\partial n(m, r, t)}{\partial t}=\frac{1}{2} \int_{0}^{m} \kappa\left(m^{\prime}, m-m^{\prime}\right) n\left(m^{\prime}, r, t\right) n\left(m-m^{\prime}, r, t\right) d m^{\prime} \\
-n(m, r, t) \int_{0}^{\infty} \kappa\left(m, m^{\prime}\right) n\left(m^{\prime}, r, t\right) d m^{\prime}-\nabla \cdot F
\end{gathered}
$$

where $\kappa\left(m, m^{\prime}\right)=\gamma P\left(m, m^{\prime}\right)$ is the collision kernel, giving the probability that a grain of mass $m$ will 
collide with and stick to a grain of mass $m^{\prime}$, where $P\left(m, m^{\prime}\right)$ is the collision probability. We set the sticking coefficient, $\gamma$, to be unity so whenever two grains collide they stick. The first integral on the right hand side equals the rate of formation of grains of mass $m$ by collisions between a grain of mass $m^{\prime}$ and a grain of mass $m-m^{\prime}$. The second integral is the rate of removal of grains of mass $m$ when such a grain combines with a grain of any other mass. The term involving $F$ is the transport term. This can be either via gravitational settling through the gas or via turbulent transport if the gas is convective. The convective velocities are derived from the evolutionary model using the Mixing Length Recipe. Collisions due to the Brownian motion of the grains are included, as is the fact that larger grains sediment faster than smaller grains and can overtake them.

In convective regions, small grains can be carried with the convective eddy while large grains would not. The different relative velocities between different sized grains result in a change in the coagulation kernel. The relative velocities in the presence of convection of grains with arbitrary sizes were recently presented by Ormel and Cuzzi (2007). The code uses Ormel and Cuzzi's expressions but also includes the sedimentation speeds. The sedimentation velocity changes the boundary between large grains which are unaffected by the convective eddies and small grains that are coupled to the gas. The difference in sedimentation speed is added in quadrature to the relative velocity in the presence of convection. The grain distribution in convective regions is calculated using a simple algorithm that mimics the eddy diffusion approximation. When convection is found to affect the grain motion, i.e. (1) the grain's sedimentation speed is smaller than the speed of the eddy and (2) the grain can be entrapped in the largest eddy (whose size is defined as 1.5× the pressure scale height), grains are redistributed so that the ratio of mass density of grains to the gas' mass density is constant. The grain mass in the convective regions can then be conserved.

The density of the grains is assumed to be $2.8 \mathrm{~g} \mathrm{~cm}^{-3}$, and the initial grain size $a_{0}$ is taken to be $10^{-5}$ $\mathrm{cm}$. The grains are taken to be composed primarily of silicates. Evaporation of grains is not included in the calculation. However, evaporation of grains is negligible at the internal temperatures of our models. The initial size is not of significant importance as long as it is relatively small. The grains are initially homogeneously mixed within the protoplanet. The size distribution is divided among bins with radii $\mathrm{a}_{i}$ that are logarithmically spaced in mass. The size of a grain in bin $i, a_{i}$, is taken to be $a_{0} 2^{i / 3}$. The total number of bins is chosen to be 40 so most of the grains are accommodated and can grow to large sizes. Initially, only the smallest mass bin is populated, and as the grains collide and grow the larger size bins are filled. Since some grains could reach the largest bin and therefore can grow further, the code can add an extra bin in which the larger grains are accumulated. At the end of the time step the largest grains are returned to the largest bin. This procedure ensures grain mass conservation.

The timesteps of the sedimentation code are chosen to be small enough to ensure that by the end of the timestep the change in number density in each atmospheric layer, and every size bin does not exceed one 
percent. The maximum timestep is set to be $10^{6}$ seconds. More details on the numerical procedure can be found in Movshovitz et al. (2010).

The mass fraction of grains ( $1 \%$ for solar composition) is constant for a given metallicity. Since the dustto-gas ratio changes with metallicity, metal-rich protoplanets naturally have larger grain number densities. When grains manage to grow large enough they can gain high enough sedimentation speeds to settle from the radiative region and reduce the atmospheric opacity (see Podolak, 2003). At each stage of the evolution, given a planetary model, the grain opacity $\kappa$ is calculated using Mie theory (van de Hulst 1957). The terms for scattering and absorption efficiencies are summarized in Movshovitz and Podolak (2008). The evolution of the grains is calculated over a given time interval, typically 250 years, for a fixed protoplanetary structure. The grain opacity values at different atmospheric heights, at the end of the time interval, are then entered into the evolutionary model. The planetary evolution is then calculated over the same 250 -year interval, with the grain opacity a function of time, interpolated in time between the grain distributions at the beginning and end of the interval. At the end of the time interval, the new planetary structure is used to evolve the grains for another 250 years, and the process is repeated. Again, we consider protoplanets with masses of 3, 5 , and $7 \mathrm{M}_{J}$, and account for three different metallicities: solar, solar $/ 3$, and $3 \times$ solar.

We find that in all cases the opacity can be reduced substantially, leading to much shorter contraction timescales than those found in the previous section. Figure 4 presents the opacities as a function of depth in the planet at different times for 3,5 , and $7 \mathrm{M}_{J}$ with low (solar/3) and high (solar $\times 3$ ) metallicities. Grain growth and opacity reduction are most efficient when the number density of grains is large, allowing frequent collisions. As a result, opacity reduction is found to be greatest for metal-rich and massive protoplanets.

Initially, the protoplanets are found to be fully convective with thin radiative outer layers. As the evolution progresses, the grains settle out of the radiative layer and the opacity is reduced, causing an increase in luminosity. Higher luminosity increases the radiative gradient and promotes convection. The outer radiative zone gradually disappears and the object becomes fully convective, apart from the radiative surface boundary condition. The convection results in the mixing of grains back up to the surface and the opacity increases again. This process can be seen in detail in figure 4. For example, $7 \mathrm{Mj}$ with high metallicity starts with a fairly flat opacity curve, the opacity then decreases with time especially in the surface layers, but at 500 years the planet becomes fully convective and the opacity in the outer layers increases. At still later times (700 years), the outer layers become radiative again and the opacity decreases significantly. A similar behavior occurs in some of the other cases.

Figure 5 shows the grain number density as a function of grain size for a metal-poor $7 \mathrm{M}_{J}$ planet at different times, and at three different layers. At time zero only grains with sizes $a_{0}=10^{-5} \mathrm{~cm}$ exist. Since the planet contracts with time the layers are defined by the percentage of planetary mass instead of an absolute height. The first layer is taken to be the uppermost layer (the surface), at this layer the mass 
integrated outward from the center equals the planetary total mass $(\mathrm{m}=100 \%)$. We present two other layers defined by integrated masses of $70 \%$ and $50 \%$ of the total mass. The upper panel corresponds to the planetary surface. As can be seen from the figure, at 100 years (black dots) there is already a population of larger-size grains due to grain growth at the surface. Grain growth and settling at the surface keep removing small grains as long as the planet's surface is radiative. When the planet becomes fully convective small grains are mixed throughout the entire envelope and are carried back to the surface (red dots). As convection continues more larger grains can be carried to the surface leading to a nearly flat size distribution, although small grains are slightly depleted because they are carried to deeper layers by convection (blue dots). Once the planet's atmosphere becomes radiative again efficient grain growth and sedimentation continue with large grains settling to deeper layers. At deeper regions within the protoplanet grain sedimentation provides a source of larger grains, and in these regions the number density of large grains is actually increasing with time (see middle and bottom panels). When the envelope becomes convective the mixing of small grains leads to an increase in the number density of small sizes deeper in the envelope. The inner regions are less effected by convective mixing, and the size distributions at 600 years and 850 years (not shown) are similar. As time progresses small grains are more depleted due to continuous grain growth and settling.

Figures 6-8 present the evolutionary tracks for the three planetary masses: 3,5 and $7 \mathrm{M}_{J}$, with solar, $3 \times$ solar and, solar $/ 3$ compositions when grain growth and sedimentation are included. We find that the $3 \mathrm{M}_{J}$ protoplanet will collapse after $\sim 1,000,1600$, and 2300 years respectively, for metal-rich, solar, and metal-poor compositions. These timescales are significantly shorter compared to the ones ignoring grain coagulation and sedimentation (section 2). $5 \mathrm{M}_{J}$ protoplanets have even shorter precollapse timescales, with the shortest found for the metal-rich case for which the evolution time is found to be only 700 years. For solar and (solar/3) compositions the times are just over 1,500 years. Massive protoplanets with masses of $7 \mathrm{M}_{J}$ are found to have contraction timescales of 670,1300 , and 1280 years for metal-rich, solar and metal-poor compositions, respectively.

As can be seen from the figures, the shortest evolution is found for the metal-rich protoplanets (red curves), which is the opposite trend to that shown in Figures 1-3. Although metal-poor objects start with a lower opacity and therefore a higher luminosity, after about a thousand years the metal-rich protoplanets actually achieve lower opacity values. While metal-rich protoplanets have more grains and therefore initially have higher opacities, grain growth and settling become very efficient in their atmospheres after a relatively short time and the opacity decreases significantly. Metal-poor protoplanets on the other hand start with low opacities due to a lower dust-to-gas ratio, but grain growth and sedimentation are less efficient due to the lower number densities and less frequent collisions. The result, that metal-rich protoplanets evolve faster, can be understood by using the approximation presented in the appendix.

To test the robustness of the results we have redone the computation taking smaller time intervals for the 
opacity recalculation. Figure 9 compares the evolution of a metal-poor and metal-rich $7 \mathrm{M}_{J}$ protoplanets (solar $/ 3$ and $3 \times$ solar metallicities, respectively) with time intervals of 250 years (black, red) and 50 years (gray, pink) between opacity calculations. Time intervals shorter than 50 years between opacity calculations would already be significantly smaller than the characteristic coagulation and sedimentation timescales and could cause numerical effects. The smaller timesteps allow us to study in more detail how the opacity reduction affects the mechanism by which energy is transferred within the planet. The planetary luminosity is the physical parameter that is most affected by the opacity. Initially the luminosity remains nearly constant, as the density in the outer layers is low and there has not been enough time for significant grain settling. As the evolution progresses and opacity is reduced the luminosity increases. Once the protoplanets become fully convective small grains remix to the surface and the luminosity decreases. The resulting drop in luminosity then again produces an outer radiative zone. Once this is present the grain settling results in an increase in luminosity, and the cycle is repeated. A gradual upward trend of the average luminosity occurs near the end of the evolution because the average grain size has increased through a significant part of the mass of the outer envelope of the planet, resulting in overall lower opacity.

While the global evolution is unchanged for metal-poor $7 \mathrm{M}_{J}$, with similar temperatures and radii, there is a noticeable difference in the behavior of luminosities. The luminosity in the high-time-resolution case oscillates around the curve for the low time resolution. The pre-collapse timescale is found to change only by a small fraction, from 1336 years in the low-time-resolution case to 1278 years.

Also for $7 \mathrm{M}_{J}$ with $3 \times$ solar metallicity the physical parameter which is most affected by the opacity calculation time-intervals is the luminosity. However, unlike in the low-metal case, the total evolutionary time is found to change from 470 years to 670 years, a relatively large difference. The cause for this difference is related to the effect of opacity on the planetary energy transport mechanism. With too large time-intervals between opacity calculations transitions between convective and radiative envelopes can be skipped, resulting in different evolutionary tracks. The evolution with smaller time-intervals is therefore more accurate. Nevertheless, it should be noted that in both cases the evolution to the onset of collapse is found to be shorter than 1000 years. The solar case should be about the same as the metal-poor case due to their very similar evolutions (see figure 8).

\section{Implications on Core Formation and Accretion of Solids}

Accretion of planetesimals is most efficient when the protoplanets are extended and fill most or all of their feeding zone; planetesimals are then captured due to gas drag. Core formation is possible as long as the central temperatures are low enough to allow the existence of solid material that can settle to the center. Since both of these processes could take place mostly during the pre-collapse evolution, a change in the pre-collapse timescale will significantly impact their efficiency. 
The results presented in section 2, in which the opacity was assumed to be proportional to metallicity, suggest that the pre-collapse timescale is proportional to the planetary metallicity, so metal-rich protoplanets would have slower contraction and more time to accrete planetesimals and form cores. In this scenario, giant planets around metal-rich stars, if formed by gravitational instabilities, would be enriched with heavier elements compared to their host star, and would have larger cores than solar-composition planets. However it should be noted that if gravitational instabilities occur mostly at very large radial distances, the planetesimal accretion efficiency decreases significantly, and the protoplanets are predicted to remain with stellar compositions, at least if they have solar composition.

In paper I we present the masses of heavy elements that can be accreted by protoplanets, with solar composition and masses between 3 and $10 \mathrm{M}_{J}$, at large radial distance. The exact amount of solids that can be captured depends on the disk properties, the planetary location, and the planetesimals' sizes and velocities. For conditions similar to the ones expected for the HR 8799 planetary system, we find that a $10 \mathrm{M}_{J}$ planet could accrete between 0 and $90 \mathrm{M}_{\oplus}$ of heavy elements, if formed between 24 and $68 \mathrm{AU}$, respectively. Planets of 7 and $5 \mathrm{M}_{J}$ can capture between 0 and 46 , and 0 and $23 \mathrm{M}_{\oplus}$ of solids, at radial distances between 24 and $68 \mathrm{AU}$, respectively. A planet of $3 \mathrm{M}_{J}$, at radial distances between 24 and $68 \mathrm{AU}$, is found to accrete up to $37.5 \mathrm{M}_{\oplus}$ of heavy elements. Although in some cases a non-negligible mass of heavy elements can be accreted, the overall planetary composition remains close to stellar. But the longer evolution of metal-rich protoplanets, as just described, could lead to much more significant accretion of solids at wide orbits. On the other hand, metal-rich objects will be more vulnerable to tidal disruption and therefore may not survive. Giant protoplanets with low metallicities are likely to stay metal poor.

The picture changes significantly when grain coagulation and sedimentation are considered (section 3). In that case we find that the pre-collapse timescales can be orders of magnitude shorter. For example, for the $5 \mathrm{M}_{J}$ solar case the time scale is reduced from 26,000 to 1600 years, and for the metal-rich case the reduction is from 75,000 to 700 years. The characteristic pre-collapse timescale is found to be of the order of one thousand years. The exact numbers change with planetary mass and metallicity, but in general it is found that when grain growth and settling are included there is much less sensitively to the planetary mass and the assumed metallicity. Another result which differs considerably from the one presented in section 2 is that metal-rich planets actually have shorter contraction timescales than metal-poor or solar-composition planets, due to their efficient grain growth resulting from their original large number densities, although the difference in timescale is not always large. The results from section 3 suggest that there is not much opportunity to capture solids for a very large range of planetary masses and compositions, because of the sharply reduced time scales. To test the possibility of core formation we follow the grain settling all the way to the planetary center. We find that in all cases although there is a substantial settling in the outer layers that leads to opacity reduction, grains cannot settle to the innermost region and form cores. In this scenario 
we find that the planetary objects are likely to remain with stellar abundance and contain no cores.

Recently, Boley and Durisen (2010) have found that gravitationally unstable clumps formed in spiral arms could have enriched initial concentrations of heavy elements as well as possible concentration of solid material toward the center of the clump. This configuration might favor core formation in protoplanets at early stages of the evolution. Although in this scenario cores could be formed, more detailed calculations are needed before a robust conclusion can be reached.

\section{Summary}

We investigate the effect of opacity on the early evolution of giant gaseous protoplanets which have formed by gravitational instability in the outer regions of protoplanetary disks. First, we model the evolution of 3, 5 , and $7 \mathrm{M}_{J}$ protoplanets with different metallicities simply by scaling their grain opacity with metallicity. In that case we find that the pre-collapse timescale is proportional to planetary metallicity; metal-poor protoplanets have the short contraction timescales while metal-rich protoplanet are found to contract over significantly longer timescales. We note that the shorter pre-collapse stages of metal-poor protoplanets can support their survival. Metal-rich protoplanets on the other hand, spend longer times in the extended phase and therefore are predicted to be more vulnerable to destruction. However, if they do manage to survive, they have better opportunity to accrete solid planetesimals and form heavy-element cores.

We next investigate the effect of opacity reduction on the pre-collapse evolution due to grain coagulation and sedimentation. We find that including these processes leads to significantly shorter evolutionary timescales for all the planetary masses and metallicities considered. The pre-collapse timescale is found to be of the order of one thousand years for masses between 3 and $7 \mathrm{M}_{J}$, and is relatively insensitive to planetary composition. We find that in this scenario the pre-collapse evolution of a metal-rich protoplanet is actually shorter than that of a metal-poor planet, due to very efficient opacity reduction caused by the larger amounts of grains initially present in the atmosphere, that lead to rapid grain growth and settling. The considerably shorter pre-collapse stages found in this scenario may reduce the risk of clump disruption, and support their survival. Since clumps accrete gas (and solids) most efficiently during their early evolution, shortening the pre-collapse stage would also limit the growth of the newly-formed protoplanets and would lead to smaller final masses. On the other hand, the short pre-collapse evolution would lead to negligible enrichment with heavy elements and will suppress the formation of cores in these objects. The results for the evolutionary timescales for the two cases we consider are summarized in Table 1.

The presented work cannot provide strong predictions due to the complex nature of the problem. Here we show, in a simple one-dimensional approximation, that adding grain coagulation and settling leads to very different results from the ones obtained when these processes are not included. Although our results suggest that massive planets formed by gravitational instabilities are unlikely to be significantly metal enriched 
relative to the star, observations of giant planets with stellar compositions at the surface should not be taken as evidence for formation by gravitational instability. Massive giant planets formed by core accretion could also have a nearly stellar overall composition. In the standard core accretion model the typical core (i.e. heavy elements) masses do not exceed several Earth masses, while the rest of the planetary mass consists of residual nebular gas (Hubickyj et al., 2005; Movshovitz et al., 2010). The exact composition of the gas would depend on disk properties, orbital location, formation timescale, etc. If the composition of the residual gas is stellar, or somewhat depleted in heavy elements compared to the star, the total abundance of the forming giant planet would be about stellar. As a result, giant planets with nearly stellar compositions could be explained by both core accretion and gravitational instability.

Naturally, there are more processes that could affect the early evolution and survival of giant protoplanets. For example, ongoing accretion of nebular gas with small dust grains would increase the opacity at the outer layers resulting in less rapid contraction. In addition, accretion would affect the planetary luminosity and therefore its contraction time. Planetary rotation could also lead to slower contraction, however, the real effect of angular momentum has to be calculated in detail before a definite conclusion can be reached. Our calculation does not assume a specific location for the formation of the planet, except that it has to be outside $20 \mathrm{AU}$ for tidal stability. In actual disks protoplanets could migrate inward or outward, a process which could also affect the planets' survival.

In this work we assume that all solids are in the form of small grains. However, if a substantial fraction of the solid material were in the form of planetesimals the opacity and the timescale for evolution would be affected. In the case of no settling (case I) the evolution times would be shorter than the ones given in table 1 due to lower opacity. In case II, the evolution timescale would be somewhat longer due to slower grain growth. It is unclear whether appreciable planetesimal formation had occurred at early stages of clump formation, especially at the large radial distances which we consider (>20 AU). We have now added one missing piece to the puzzle, and hope that future investigations including additional effects will provide a clearer picture of early planetary evolution.

\section{Acknowledgments}

P. B. acknowledges support from NASA Origins grant NNX08AH82G and NSF grant AST-0908807. R. H. acknowledges support from NASA through the Southwest Research Institute.

\section{References}

Alexander, D., Ferguson, J., 1994. Low-temperature Rosseland opacities. Astrophys. J. 437, 879-891.

Bodenheimer, P., Grossman, A. S., Decampli, W. M., Marcy, G., Pollack, J. B., 1980. Calculations of the 
evolution of the giant planets. Icarus 41, 293-308.

Boley, A. C., 2009. The two modes of giant planet formation. Astrophys. J. 695, L53-56.

Boley, A. C., Hayfield, T., Mayer, L., Durisen, R. H., 2010. Clumps in the outer disk by disk instability: Why they are initially gas giants and the legacy of disruption. Icarus, 207, 509-516.

Boley, A. C and Durisen, R. H., 2010. Enrichment and Differentiation in Gas Giants During Birth by Disk Instability. arXiv:1005.2624

Boss, A. P., 1997. Giant planet formation by gravitational instability. Science 276, 1836-1839.

Boss, A. P., 2002. Evolution of the solar nebula. V. Disk instabilities with varied thermodynamics. Astrophys. J. 576, 462-472.

Boss, A. P., 2008. Flux-limited diffusion approximation models of giant planet formation. Astrophys. J. 677, 607-615.

Cai, K., Durisen, R. H., Michael, S., Boley, A. C., Meja, A. C., Pickett, M. K., D'Alessio, P. The Effects of Metallicity and Grain Size on Gravitational Instabilities in Protoplanetary Disks. 2006a, ApJ, 636, L149L152.

Cai, K., Durisen, R. H., Michael, S., Boley, A. C., Meja, A. C., Pickett, M. K., D’Alessio, P. Erratum: "The Effects of Metallicity and Grain Size on Gravitational Instabilities in Protoplanetary Disks", 2006b, ApJ, 642, L173-L173.

Cai, K., Pickett, M. K., Durisen, R. H., Milne, A. M., 2009. Giant planet formation by disk instability: A comparison simulation with an improved radiative scheme. Astrophys. J. Letters, submitted. arXiv:0907.4213

Cameron, A. G. W., 1978. Physics of the primitive solar nebula and of giant gaseous protoplanets. In: Gehrels, T. (Ed.) Protostars and planets: Studies of star formation and of the origin of the solar system. University of Arizona Press, Tucson, pp. 453-487.

Decampli, W. M., Cameron, A. G. W., 1979. Structure and evolution of isolated giant gaseous protoplanets. Icarus 38, 367-391.

Dodson-Robinson, S., Veras, D., Ford, E., Beichman, C. A., 2009. The formation mechanism of gas giants on wide orbits. Astrophys. J. 707, 79-88.

Durisen, R. H., Boss, A. P., Mayer, L., Nelson, A. F., Quinn, T., Rice, W. K. M. , 2007. Gravitational instabilities in protoplanetary disks and implications for giant planet formation. In Reipurth, B. et al. (Eds.), Protostars and Planets V, Univ. of Arizona Press, Tucson, pp. 607-622.

Gammie, C. F., 2001. Non-linear outcome of gravitational instability in cooling, gaseous disks. Astrophys. J. 553, 174-183.

Helled, R., Podolak, M., Kovetz, A., 2006. Planetesimal capture in the disk instability model. Icarus 185, $64-71$. 
Helled, R., Podolak, M., Kovetz, A., 2008. Grain sedimentation in a giant gaseous protoplanet. Icarus 195, 863-870.

Helled, R., Bodenheimer, P., 2010. Metallicity of the massive protoplanets around HR 8799 if formed by gravitational instability. Icarus, 207, 503-508 (paper I).

Hubickyj, O., Bodenheimer, P., Lissauer, J. J., 2005. Accretion of the gaseous envelope of Jupiter around a 5-10 Earth-mass core. Icarus 179, 415-431.

Kalas, P. et al., 2008. Optical imaging of an exosolar planet 25 light years from Earth. Science 322, 1345-47. Marois, C., Macintosh, B., Barman, T., Zuckerman, B., Song, I., Patience, J., Lafreniere, D., Doyon, R., 2008. Direct imaging of multiple planets orbiting the star HR 8799. Science 322, 1348-1352.

Mayer, L., Lufkin, G., Quinn, T., Wadsley, J., 2007. Fragmentation of gravitationally unstable gaseous protoplanetary disks with radiative transfer. Astrophys. J. 661, L77-L80.

Meru, F., Bate, M. R., 2010. Exploring the conditions required to form giant planets via gravitational instability in massive protoplanetary disks. arXiv:1004.3766.

Movshovitz, N., Podolak, M., 2008. The opacity of grains in protoplanetary atmospheres, Icarus, 194, 368378.

Movshovitz, N., Bodenheimer P., Podolak, M., Lissauer, J. J., 2010. Formation of Jupiter with opacities based on detailed grain physics, Icarus, in press. arXiv:1005.3875

Ormel, C. W., Cuzzi, J. N., 2007. Closed-form expressions for particle relative velocities induced by turbulence. Astron. Astrophys, 466, 413-420.

Podolak, M., 2003. The contribution of small grains to the opacity of protoplanetary atmospheres. Icarus 73, 163-179.

Pollack, J. B., Hubickyj, O., Bodenheimer, P., Lissauer, J. J., Podolak, M., Greenzweig, Y., 1996. Formation of the giant planets by concurrent accretion of solids and gas. Icarus 124, 62-85.

Pollack, J., McKay, C., Christofferson, B., 1985. A calculation of the Rosseland mean opacity of dust grains in primordial Solar System nebulae. Icarus 64, 471-492.

Rafikov, R. R., 2007. Convective cooling and fragmentation of gravitationally unstable disks. Astrophys. J. $662,642-650$.

Rafikov, R. R., 2009. Properties of gravitoturbulent accretion disks. Astrophys. J. 704, 281-291. van de Hulst, H. C., 1957. Light scattering by small particles. John Wiley \& Sons, New York. 


\section{Appendix 1: A simple approximation for grain growth timescale}

The coagulation time can be estimated by dividing the mean free path for grain-grain collisions by the random (thermal) speed of the grains. Taking $X$ to be the mass fraction of dust in the gas (changing with metallicity), the total mass of grains within the body is given by $M_{\text {grain }}=X M_{\text {gas }}$, where $M_{\text {gas }}$ is the mass of the gas. Assuming that all the grains within the protoplanet have a similar size, $a_{0}$, the number density of grains can be written as

$$
n_{\text {grain }}=\frac{3 X \rho}{4 \pi \rho_{\text {grain }} a_{0}^{3}}
$$

with $\rho$ and $\rho_{\text {grain }}$ being the density of the planetary envelope and the grain, respectively.

The cross section of the grains is given by $\sigma=4 \pi a_{0}^{2}$, and the mean free path between collisions is

$$
\lambda=\frac{1}{\sqrt{2} n \sigma}=\frac{\rho_{\text {grain }} a_{0}}{3 \sqrt{2} X \rho} .
$$

The velocity of the grains is taken to be the thermal velocity $v_{t h}$ is given by

$$
v_{t h}=\sqrt{\frac{8 k T}{\pi m}}
$$

where $T$ is the temperature of the atmosphere, $k$ is Boltzmann's constant, and $m$ being the grain's mass. Finally, the time between collisions is given by

$$
\tau_{\text {coag }}=\frac{\lambda}{v_{\text {th }}}=\frac{\rho_{\text {grain }} a_{0}}{3 \sqrt{2} X \rho} \sqrt{\frac{\pi m_{\text {grain }}}{8 k T}}=\frac{\pi}{6 X \rho} \sqrt{\frac{\rho_{\text {grain }}^{3} a_{0}^{5}}{3 k T}} .
$$

Assuming that the atmospheric properties are about the same for protoplanets with different metallicities, one can see from equation (5) that the coagulation time is inversely proportional to $X$, the dust-to-gas ratio. As a result, grain growth (followed by grain settling) is more efficient in metal-rich protoplanets. This is only a rough estimate for the coagulation time, since it does not include growth in which a grain with higher sedimentation velocity overtakes grains with smaller sedimentation speeds, a mechanism that can be very efficient especially for small grain sizes. Once a slightly bigger grain is formed, it can immediately sweep up the small grains in the background. The approximation above is valid only for a radiative envelope, however in the models considered here the opacity in the outer radiative envelope essentially determines the time scale of evolution. The coagulation time in the outer layers of these models at the early stages of evolution is only a few years for $a_{0}=10^{-5} \mathrm{~cm}$. 


\begin{tabular}{lccc}
\hline & \multicolumn{3}{c}{ Planetary Composition } \\
Planetary Mass & solar $/ 3$ & solar & $3 \times$ solar \\
\hline $3 \mathrm{M}_{J}-$ case I & $29,075 \mathrm{yrs}$ & $78,189 \mathrm{yrs}$ & $223,705 \mathrm{yrs}$ \\
$3 \mathrm{M}_{J}$ - case II & $2,337 \mathrm{yrs}$ & $1,667 \mathrm{yrs}$ & $1,062 \mathrm{yrs}$ \\
\hline $5 \mathrm{M}_{J}$ - case I & $9,119 \mathrm{yrs}$ & $26,160 \mathrm{yrs}$ & $75,379 \mathrm{yrs}$ \\
$5 \mathrm{M}_{J}$ - case II & $1,707 \mathrm{yrs}$ & $1,587 \mathrm{yrs}$ & $720 \mathrm{yrs}$ \\
\hline $7 \mathrm{M}_{J}$ - case I & $3,880 \mathrm{yrs}$ & $11,770 \mathrm{yrs}$ & $34,840 \mathrm{yrs}$ \\
$7 \mathrm{M}_{J}$ - case II & $1,336 \mathrm{yrs}$ & $1,304 \mathrm{yrs}$ & $474 \mathrm{yrs}$ \\
\hline
\end{tabular}

Table 1: Pre-collapse evolution timescales for the two different cases considered: Case I: the opacity is scaled with planetary metallicity and is constant with time. Case II: the opacity can be reduced with time as a result of grain growth and settling.

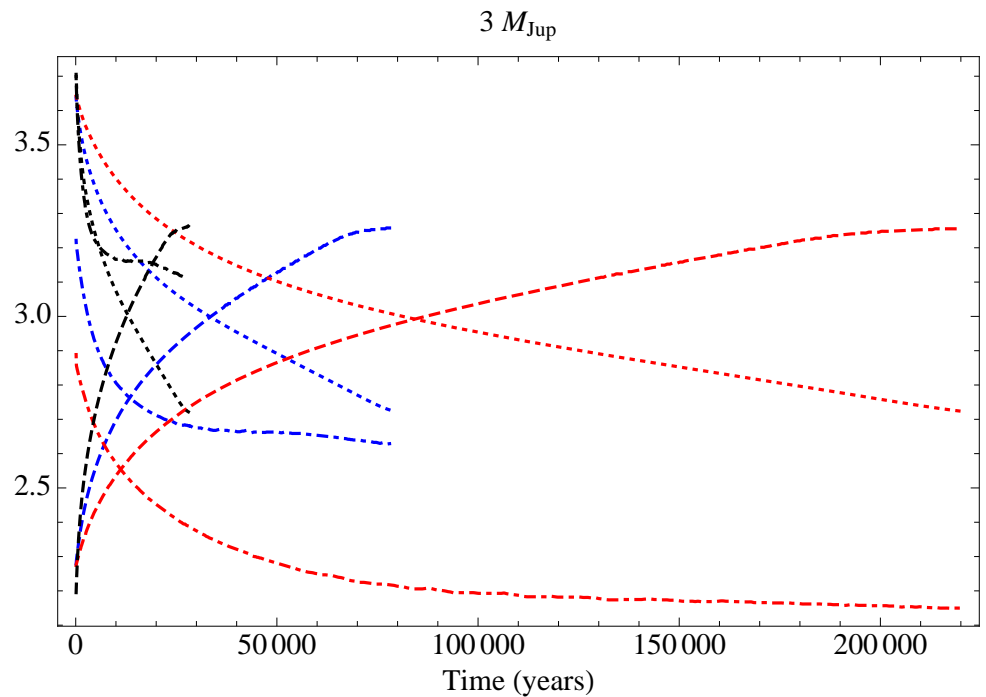

Figure 1: Physical properties as a function of time for 3 Jupiter mass protoplanets with solar (blue), three times solar (red) and solar/3 (black) compositions. The dashed, dotted, and dot-dashed curves represent $\log \left(\mathrm{T}_{\mathrm{c}}\right)[\mathrm{K}], \log \left(\mathrm{R} / \mathrm{R}_{\mathrm{Jup}}\right)[\mathrm{cm}]$, and $\log \left(\mathrm{L} / 10^{26}\right)\left[\mathrm{erg} \mathrm{s}^{-1}\right]$, respectively. 


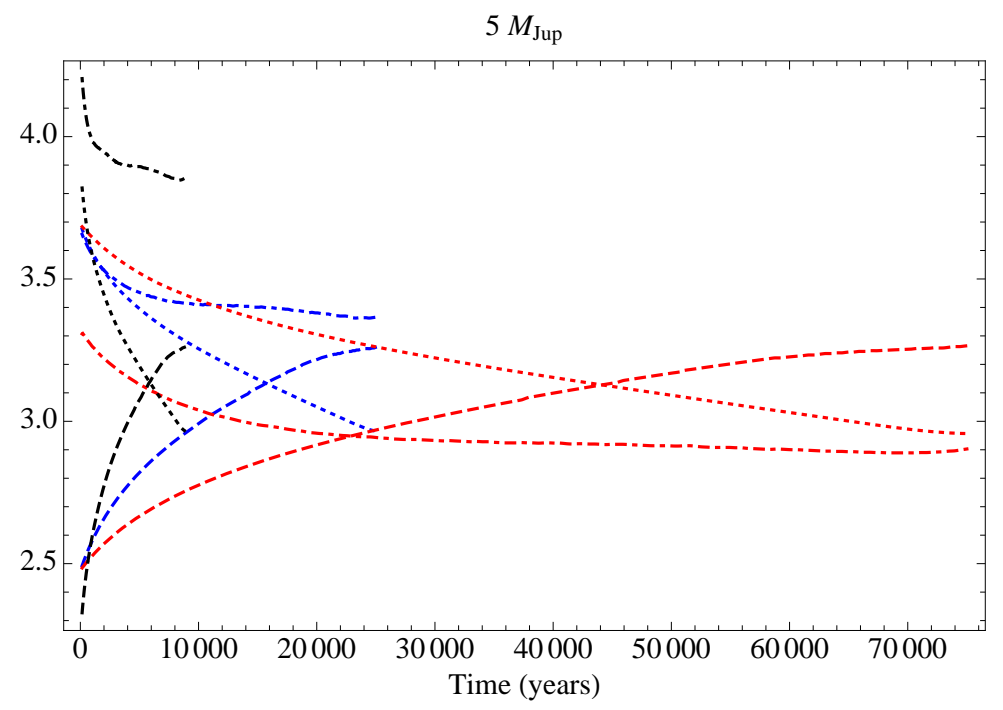

Figure 2: Physical properties as a function of time for 5 Jupiter mass protoplanets with solar (blue), three times solar (red) and solar/3 (black) compositions. The dashed, dotted, and dot-dashed curves represent $\log \left(\mathrm{T}_{\mathrm{c}}\right)[\mathrm{K}], \log \left(\mathrm{R} / \mathrm{R}_{\mathrm{Jup}}\right)[\mathrm{cm}]$, and $\log \left(\mathrm{L} / 10^{26}\right)\left[\mathrm{erg} \mathrm{s}^{-1}\right]$, respectively.

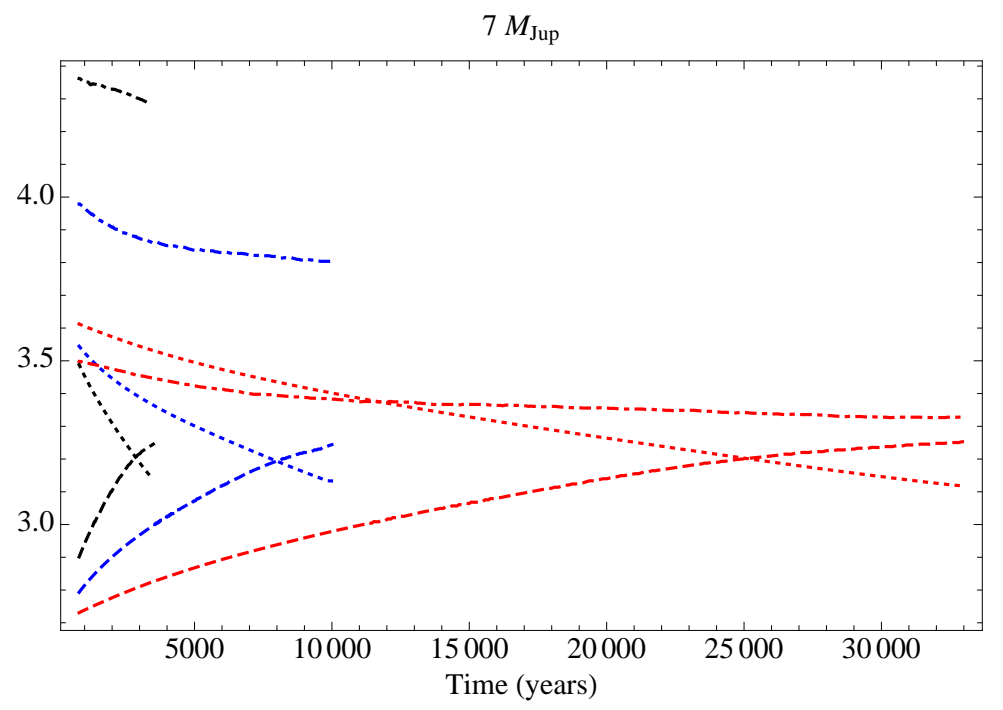

Figure 3: Physical properties as a function of time for 7 Jupiter mass protoplanets with solar (blue), three times solar (red) and solar/3 (black) compositions. The dashed, dotted, and dot-dashed curves represent $\log \left(\mathrm{T}_{\mathrm{c}}\right)[\mathrm{K}], \log \left(\mathrm{R} / \mathrm{R}_{\mathrm{Jup}}\right)[\mathrm{cm}]$, and $\log \left(\mathrm{L} / 10^{26}\right)\left[\mathrm{erg} \mathrm{s}^{-1}\right]$, respectively. 

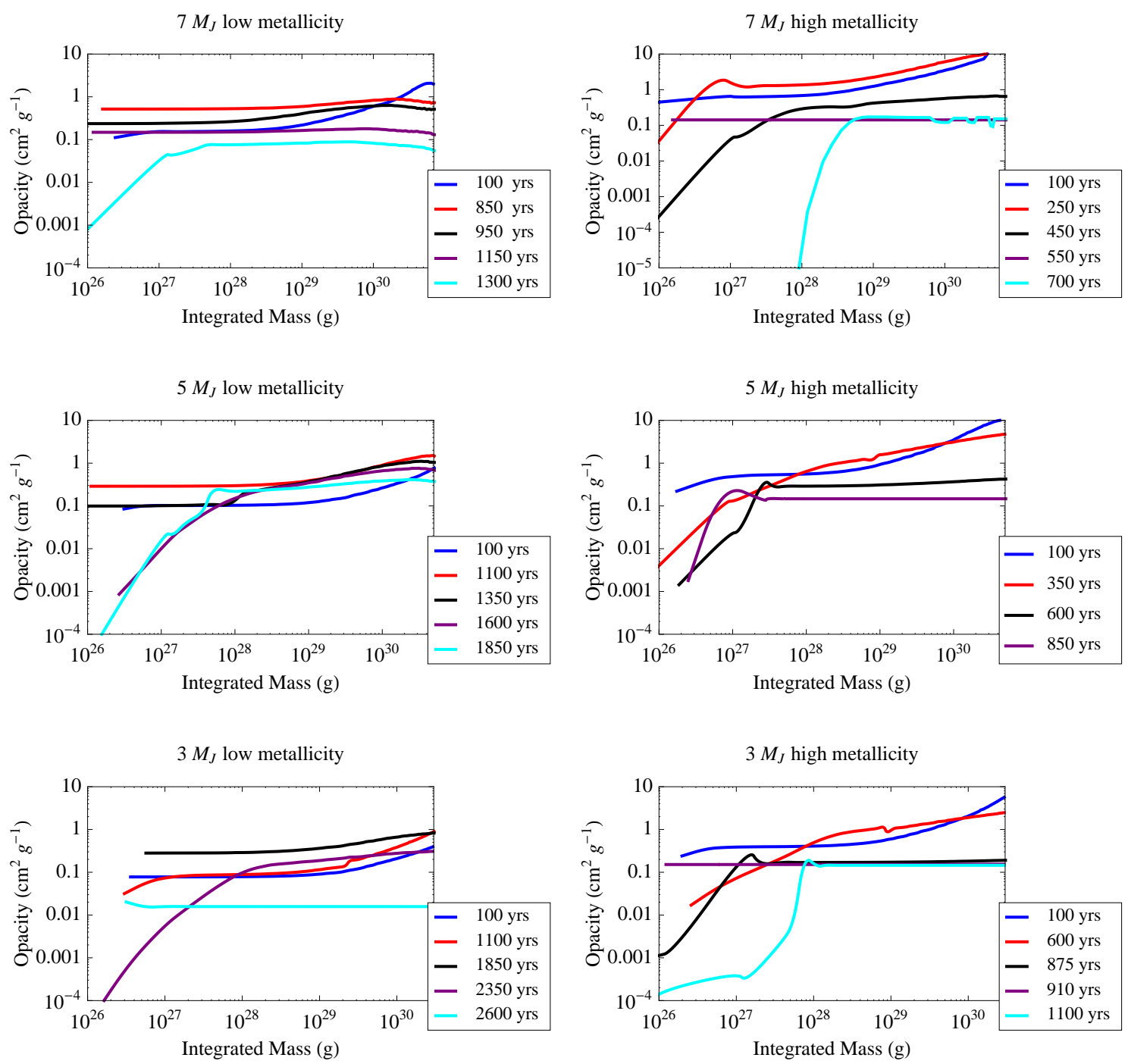

Figure 4: Rosseland mean opacity as a function of depth in the protoplanetary envelope at various times when grain coagulation and sedimentation are included. The left-hand panels correspond to a metal abundance of $1 / 3$ solar; the right-hand panels to 3 times solar. The abscissa refers to the mass integrated inward from the surface. 

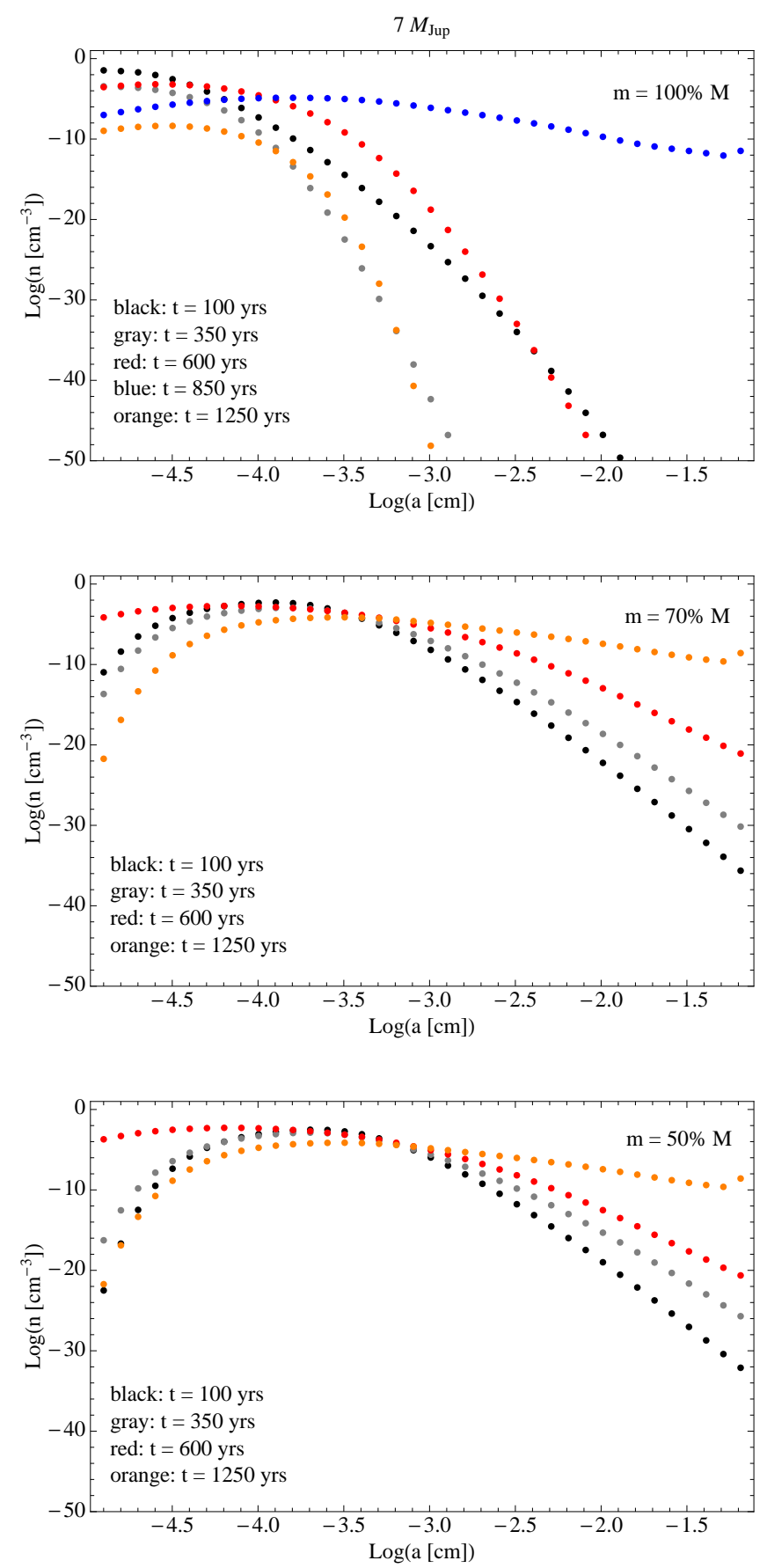

Figure 5: Grain number density 'n' vs. grain size 'a' for metal-poor $7 \mathrm{M}_{J}$ planet at different times. The three different figures correspond to three different layers in the envelope defined by the integrated mass outward from the planetary center. The figures are presented for integrated masses of 100 (top), 70 (middle), and 50 (bottom) percent of the total planetary mass. Different colors correspond to different times. 


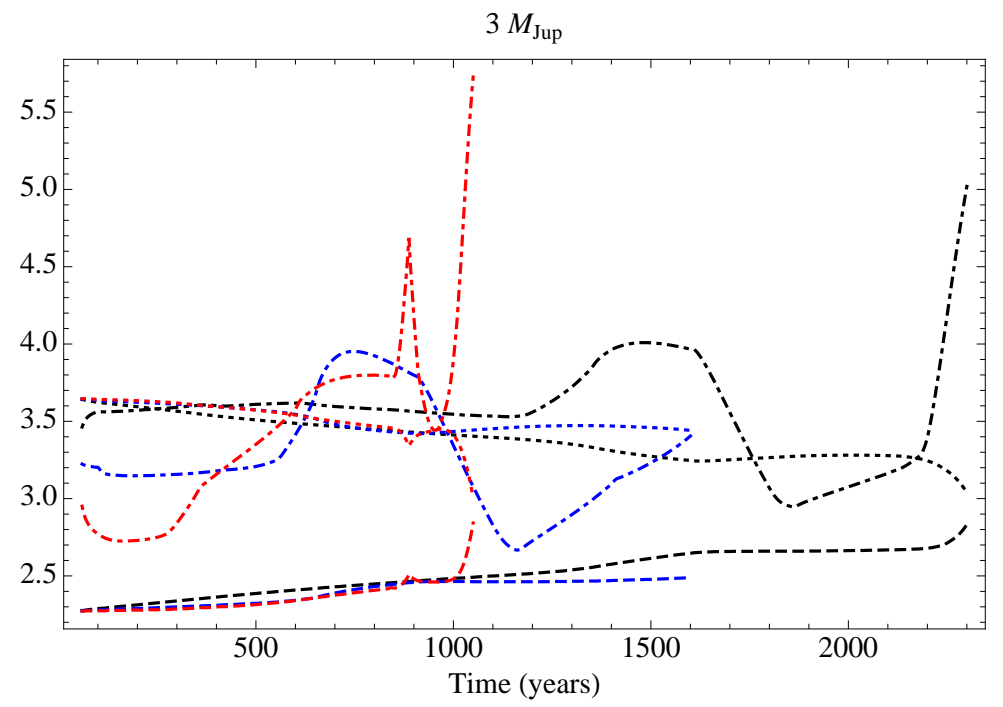

Figure 6: Physical properties as a function of time when grain growth and settling are included for 3 Jupiter mass protoplanets with solar (blue), three times solar (red) and solar/3 (black) compositions. The dashed, dotted, and dot-dashed curves represent $\log \left(\mathrm{T}_{\mathrm{c}}\right)[\mathrm{K}], \log \left(\mathrm{R} / \mathrm{R}_{\mathrm{Jup}}\right)[\mathrm{cm}]$, and $\log \left(\mathrm{L} / 10^{26}\right)\left[\mathrm{erg} \mathrm{s}^{-1}\right]$, respectively.

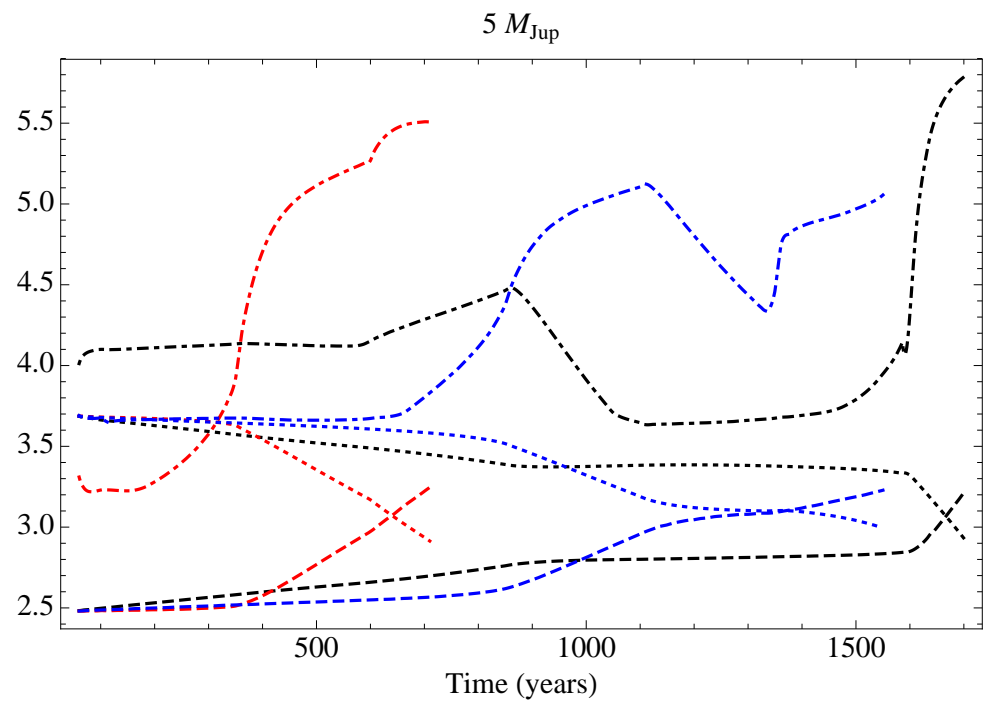

Figure 7: Physical properties as a function of time when grain growth and settling are included for 5 Jupiter mass protoplanets with solar (blue), three times solar (red) and solar/3 (black) compositions. The dashed, dotted, and dot-dashed curves represent $\log \left(\mathrm{T}_{\mathrm{c}}\right)[\mathrm{K}], \log \left(\mathrm{R} / \mathrm{R}_{\mathrm{Jup}}\right)[\mathrm{cm}]$, and $\log \left(\mathrm{L} / 10^{26}\right)\left[\mathrm{erg} \mathrm{s}^{-1}\right]$, respectively. 


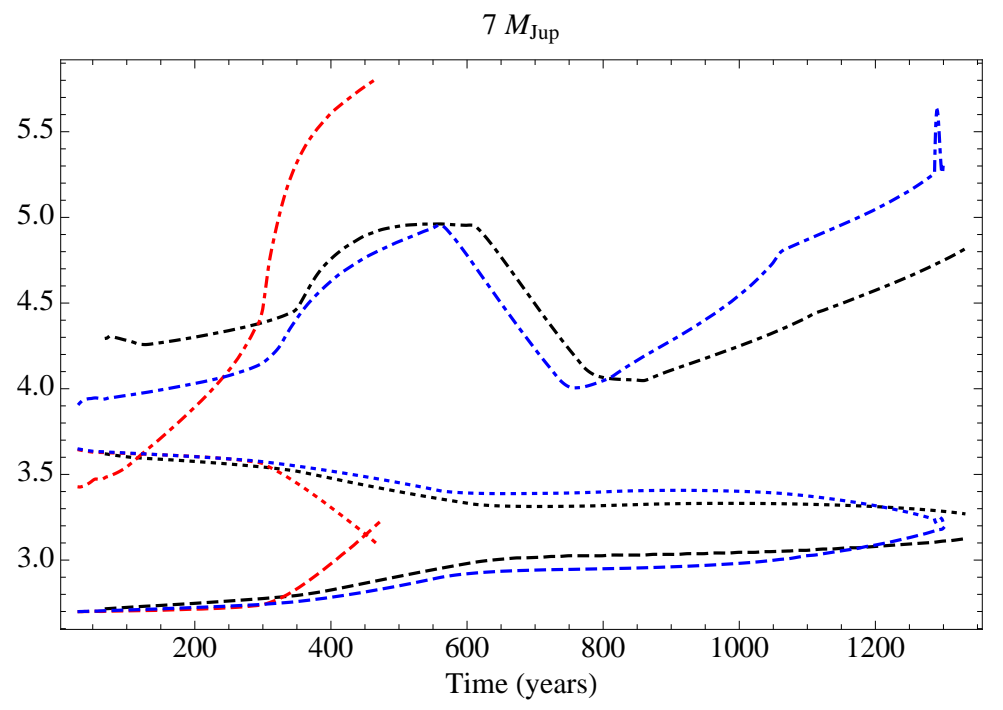

Figure 8: Physical properties as a function of time when grain growth and settling are included for 7 Jupiter mass protoplanets with solar (blue), three times solar (red) and solar/3 (black) compositions. The dashed, dotted, and dot-dashed curves represent $\log \left(\mathrm{T}_{\mathrm{c}}\right)[\mathrm{K}], \log \left(\mathrm{R} / \mathrm{R}_{\mathrm{Jup}}\right)[\mathrm{cm}]$, and $\log \left(\mathrm{L} / 10^{26}\right)\left[\mathrm{erg} \mathrm{s}^{-1}\right]$, respectively. 

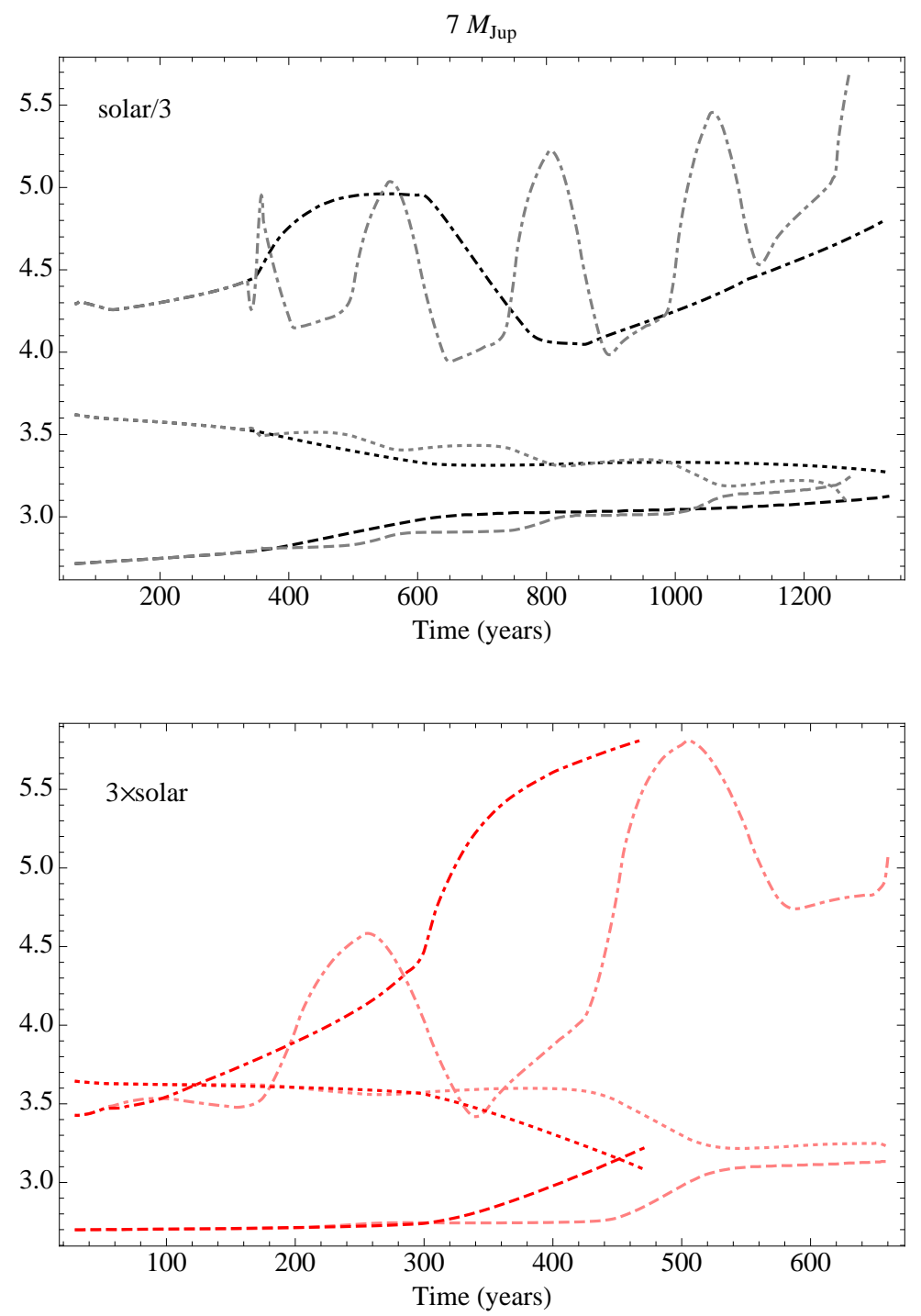

Figure 9: Physical properties as a function of time when grain growth and settling are included for $7 \mathrm{M}_{J}$ protoplanets with solar $/ 3$ (top) and $3 \times$ solar (bottom) compositions with long (black, red; 250 years) and short (gray, pink; 50 years) time intervals between opacity recalculations. Again, the dashed, dotted, and dot-dashed curves represent $\log \left(\mathrm{T}_{\mathrm{c}}\right)[\mathrm{K}], \log \left(\mathrm{R} / \mathrm{R}_{\mathrm{Jup}}\right)[\mathrm{cm}]$, and $\log \left(\mathrm{L} / 10^{26}\right)\left[\mathrm{erg} \mathrm{s}^{-1}\right]$, respectively. 